\title{
Advances in PET/CT in pediatric oncology
}

\author{
Michael J. Gelfand • Susan E. Sharp
}

Received: 12 January 2011 / Accepted: 17 January 2011

(C) Springer-Verlag 2011

Positron emission tomography (PET) has transformed nuclear medicine imaging technology. PET is an inherently tomographic technique that utilizes the simultaneous detection of 180-degree opposed $511 \mathrm{keV}$ photons emitted by positron annihilation. Collimation can be eliminated, greatly improving sensitivity and spatial resolution. By placing PET and CT scanners on the same gantry, co-registered PET/CT images can be obtained, allowing normal and abnormal physiology to be accurately depicted in a high-resolution spatial context. The CT part of the PET/CT scanner can be used in any of three modes: as a diagnostic CT scanner at appropriate diagnostic settings for pediatric $\mathrm{CT}$, at reduced non-diagnostic localization settings, and at extremely low non-imaging settings for attenuation correction only. PET/CT has improved the accuracy of PET imaging in 10-30\% of patients in adult and pediatric studies. With precise localization of PET abnormalities on PET/CT images and the anatomical information obtained from the co-registered diagnostic or localization CT, interpretative errors are reduced in frequency.

\section{Radiopharmaceuticals}

Almost all PET and PET/CT scans are performed with [F-18] 2-fluoro-2-deoxyglucose (FDG), a glucose analog that has turned out to be an excellent all-purpose radiopharmaceutical in oncology. The vast majority of non-CNS pediatric and adolescent tumors express increased glucose metabolism,

Disclaimer The authors have no financial interests, investigational or off-label uses to disclose.

M. J. Gelfand $(\bowtie) \cdot$ S. E. Sharp

Cincinnati Children's Hospital Medical Center,

Cincinnati, OH 45215, USA

e-mail: michael.gelfand@cchmc.org which is easily demonstrated on FDG PET imaging. Exceptions include hepatocellular carcinoma and renal cell carcinoma, where high levels of FDG uptake are seen in only about half of patients.

Amino acid agents, such as C-11-methionine, have been used in some centers for brain tumor imaging and are further discussed in the brain tumor section below. [F-18] fluoride was the original bone imaging agent and is now being used for bone PET as described in the bone imaging section below.

The 1.8-h half-life of F-18 allows radiopharmaceuticals such as [F-18] FDG to be synthesized in excess and distributed to most pediatric care facilities by surface or air transportation. C-11-labeled radiopharmaceuticals are limited in availability, because the short half-life of C-11 (t $1 / 2=20 \mathrm{~min}$ ) requires that a cyclotron and positron radiochemistry facility be located within a few minutes of the location where the radiopharmaceutical will be used. FDG uptake is usually quantified by computation of standardized uptake value (SUV), a measurement of fractional uptake of the radiopharmaceutical dose. SUV is usually expressed as the maximum SUV within a 3-dimensional region of interest that contains the tumor.

\section{Artifacts and false-positive studies}

Although some early series reported moderate numbers of false-positive studies, identification and prevention of artifacts has significantly reduced the frequency of unrecognized false-positive studies.

Image interpretation in lymphoma and some other malignancies might be complicated by FDG uptake in brown adipose tissue in the neck, supraclavicular regions, axillae, and mediastinum. Cold-induced FDG uptake in 
brown adipose tissue has been a particular problem in pediatric and adolescent patients, interfering with image interpretation in $25-30 \%$ of cases. Prevention of cold-induced FDG uptake in brown adipose tissue has been shown to minimize this problem. Pre-warming and, in some hospitals, medication given prior to radiopharmaceutical administration can reduce interfering FDG uptake in brown adipose tissue in $5-10 \%$ of cases. When brown adipose tissue FDG uptake is present, co-registered and fused CT images can be used to differentiate uptake in lymph nodes from uptake in areas of fat density. Use of co-registered CT images also facilitates identification of lesions, such as nonossifying fibromas, which might demonstrate focal FDG uptake.

Accurate attenuation correction in the lower chest and upper abdomen requires the position of the diaphragm to be nearly identical during PET and $\mathrm{CT}$ acquisitions. To prevent significant attenuation-correction artifacts near the diaphragm, the CT part of the PET/CT study needs to be acquired during quiet tidal respiration or at end-tidal expiration. A full inspiration diagnostic chest CT scan must be obtained separately when needed for cancer diagnosis.

\section{A paradigm for tumor imaging with radiopharmaceuticals}

Tumor imaging with radiopharmaceuticals, including PET radiopharmaceuticals and some single photon agents such as I-123-MIBG, is often performed using the following paradigm:

(1) An initial study at diagnosis to confirm radiopharmaceutical concentration in tumor, measure avidity of tumor uptake, locate metastases and, if needed, to identify the best site for biopsy.

(2) Follow-up studies at appropriate intervals during therapy to monitor the response of the tumor to therapy (by disappearance or reduction of abnormal FDG uptake).

(3) Follow-up studies at the end of therapy to locate any residual tumor that might need further therapy.

(4) Follow-up studies after completion of therapy, to monitor for tumor recurrence.

Therapeutic protocols often incorporate PET imaging at intervals that reflect this paradigm.

\section{Lymphoma}

Areas of lymphomatous involvement are consistently FDG avid, allowing disease assessment before, during and after chemotherapy. In Hodgkin lymphoma, FDG PET is typically used at diagnosis, after one or two cycles of multi-drug chemotherapy to evaluate early chemotherapeutic response, and subsequently one or two additional times, with a final scan at the end of chemotherapy. FDG uptake will usually disappear after the first or second cycle of chemotherapy. Conventional cross-sectional imaging frequently underestimates tumor response to chemotherapy, as CT evidence of tumor tends to persist with incomplete tumor shrinkage.

Five-year survival rates in Hodgkin lymphoma are high, approximately $90 \%$. Persistent FDG uptake in tumor has been shown to be a better predictor of which patients will relapse or fail initial chemotherapy than has persistent evidence of tumor on CT. The excellent 5-year survival rates after chemotherapy are tempered by $10-20 \%$ rates of second malignancy in patients who receive local radiotherapy. A recent attempt to use CT response after chemotherapy to determine the need for radiotherapy resulted in an increased rate of relapse.

Current chemotherapy protocols are evaluating whether the time of FDG disappearance can be used to modify therapy for individual patients, optimizing the duration of chemotherapy and aiding in the determination of which patients require local radiotherapy. In general, in Hodgkin disease, chemotherapy after relapse is not as effective as the initial chemotherapy; therefore, an increased rate of relapse is generally not an acceptable outcome.

In most forms of non-Hodgkin lymphoma, FDG PET is used in a similar manner. Five-year event-free survival rates are good, but not as high as in Hodgkin lymphoma.

\section{Neuroblastoma}

During the last two decades, I-123-MIBG has been the preferred radiopharmaceutical for neuroblastoma imaging. When FDG PET became available, its utility in neuroblastoma imaging was investigated with comparison to I-123-MIBG. According to data from a study combining the experiences of St. Jude Children's Research Hospital and our hospital, FDG PET is superior for imaging low-stage neuroblastoma patients in whom MIBG uptake might be low level or sometimes absent. In higher-stage neuroblastoma, MIBG is better than FDG for identifying bone or bone marrow involvement and for following response to chemotherapy. In patients undergoing chemotherapy, abnormal FDG uptake in bone or bone marrow metastases is sometimes hidden by intense FDG uptake in marrow caused by cell-stimulating factors such as granulocyte cell-stimulating factor (GCSF). However, disease in the trunk, including primary tumors and metastatic lymph nodes, is better seen on FDG PET, often because of the improved spatial resolution provided by this inherently tomographic technique. Disease stage and therapeutic history must be taken into account when choosing a functional imaging agent for individual neuroblastoma patients. 
Sarcomas

Avid FDG uptake is seen in almost all bone sarcomas, particularly osteogenic sarcoma and Ewing sarcoma. Patients are at greater risk for relapse if the maximum SUV in tumor is high at diagnosis and if the maximum SUV does not fall to 2.5 or less after initial chemotherapy. However, despite the differences in survival curves, FDG has limited predictive value in the outcomes of individual patients.

After limb salvage procedures, bulky metal endoprostheses cause extensive artifacts on CT and MRI imaging. FDG PET imaging is not significantly degraded by the endoprostheses, despite artifacts on the CT scan used for attenuation correction. Local recurrences are usually well detected on FDG PET. Prosthesis fracture and loosening are best detected by plain radiographs, but other complications in soft tissue are often detected first by FDG PET. In soft-tissue sarcomas, such as rhabdomyosarcoma, residual and recurrent tumor and new metastatic disease can be detected with FDG PET/CT.

\section{Langerhans cell histiocytosis ( $\mathrm{LCH})$}

Plain radiographs, CT, MRI and bone scintigraphy demonstrate bone destruction and healing, while FDG PET demonstrates the increased glucose metabolism associated with the proliferative activity of LCH. Early assessment of treatment response is therefore possible with FDG PET. FDG uptake disappears with successful LCH treatment months prior to the bone healing seen on the other modalities. Because $\mathrm{LCH}$ after infancy is associated with excellent survival, radiation dose reduction is important and dose reduction methods should be used when they do not compromise accuracy of diagnosis. The typical administered activity of $5.2 \mathrm{MBq} / \mathrm{kg}(0.14 \mathrm{mCi} / \mathrm{kg})$ can be reduced by $30-55 \%$ in pre-adolescent $\mathrm{LCH}$ patients according to North American Consensus Guidelines and the European Association of Nuclear Medicine Paediatric Dose Card, when 3-D PET scanners are used. CT doses can also be reduced in these pre-adolescent $\mathrm{LCH}$ patients.

Brain tumors

The high rate of glucose metabolism by normal brain tissue limits detectability of low-grade brain tumors with FDG PET. However, recent work demonstrates improved quantitative characterization of FDG uptake in high-grade gliomas.

The amino acid C-11-methionine has been used to detect low-grade brain lesions that were difficult to characterize by other methods. When C-11-methionine amino acid imaging is not available, early post-injection imaging with [F-18]3,4-dihydroxy-6-fluoro-phenylalanine (FDOPA) might be a substitute.

C-11-methionine has been shown to be useful in brain tumors for improving delineation of tumor margins prior to resection. Because of the 20 -min half-life of $\mathrm{C}-11$, this radiopharmaceutical has been used in only a few centers. In the center with the largest experience in children, use of C-11-methionine (and to a lesser extent FDG) to determine the resection margins of infiltrative high-grade tumors improved survival compared to use of MRI alone. C-11-methionine and FDG imaging were also thought to improve the choice of stereotactic biopsy sites.

\section{Bone imaging}

[F-18]fluoride was the original bone imaging agent. It disappeared from use when the Tc-99m-labeled diphosphonates became available. Now using the greatly improved sensitivity and spatial resolution of PET imaging, [F-18]fluoride bone PET provides images that are far superior to those obtained with Tc-99m-medronate. The improved image quality of [F-18]fluoride bone PET has been associated with an increased sensitivity for the detection of bone metastases in adults, but these observations have not yet been extended to children. 